\title{
Development of Machine Learning Methods in Hybrid Energy Storage Systems in Electric Vehicles
}

\author{
Tzu-Chia Chen, ${ }^{1}$ Fouad Jameel Ibrahim Alazzawi, ${ }^{2}$ John William Grimaldo Guerrero, ${ }^{3}$ \\ Paitoon Chetthamrongchai, ${ }^{4}$ Aleksei Dorofeev, ${ }^{5}$ Aras masood Ismael, ${ }^{6}$ \\ Alim Al Ayub Ahmed (D, ${ }^{7}$ Ravil Akhmadeev, ${ }^{8}$ Asslia Johar Latipah, \\ and Hussein Mohammed Esmail Abu Al-Rejal $\mathbb{D}^{10}$
}

\footnotetext{
${ }^{1}$ Department of Industrial Engineering and Management, Ming Chi University of Technology, New Taipei City 24301, Taiwan

${ }^{2}$ Department of Computer Engineering, Al-Rafidain University College, Baghdad, Iraq

${ }^{3}$ Department of Energy, Universidad de la Costa, Barranquilla, Colombia

${ }^{4}$ Faculty of Business Administration, Kasetsart University, Thailand

${ }^{5}$ I.M. Sechenov First Moscow State Medical University (Sechenov University), Department of Propaedeutics of Dental Diseases, Moscow, Russia

${ }^{6}$ Sulaimani Polytechnic University, Technical College of Informatics, Information Technology Department, Sulaymaniyah, Iraq ${ }^{7}$ School of Accounting, Jiujiang University, 551 Qianjindonglu, Jiujiang, Jiangxi, China

${ }^{8}$ British Doctor of Philosophy Degree (PhD) Standard, Associate Professor, Plekhanov Russian University of Economics (PRUE), Stremyanny Lane 36, 117997 Moscow, Russia

${ }^{9}$ Department of Informatics, Faculty of Science and Technology, Universitas Muhammadiyah Kalimantan Timur, Samarinda 75124, Indonesia

${ }^{10}$ Hodeidah University, Al Hudaydah, Yemen
}

Correspondence should be addressed to Hussein Mohammed Esmail Abu Al-Rejal; drhusseinabualrejal@gmail.com

Received 27 September 2021; Revised 3 December 2021; Accepted 16 December 2021; Published 15 January 2022

Academic Editor: Alireza Goli

Copyright (c) 2022 Tzu-Chia Chen et al. This is an open access article distributed under the Creative Commons Attribution License, which permits unrestricted use, distribution, and reproduction in any medium, provided the original work is properly cited.

The hybrid energy storage systems are a practical tool to solve the issues in single energy storage systems in terms of specific power supply and high specific energy. These systems are especially applicable in electric and hybrid vehicles. Applying a dynamic and coherent strategy plays a key role in managing a hybrid energy storage system. The data obtained while driving and information collected from energy storage systems can be used to analyze the performance of the provided energy management method. Most existing energy management models follow predetermined rules that are unsuitable for vehicles moving in different modes and conditions. Therefore, it is so advantageous to provide an energy management system that can learn from the environment and the driving cycle and send the needed data to a control system for optimal management. In this research, the machine learning method and its application in increasing the efficiency of a hybrid energy storage management system are applied. In this regard, the energy management system is designed based on machine learning methods so that the system can learn to take the necessary actions in different situations directly and without the use of predicted select and run the predefined rules. The advantage of this method is accurate and effective control with high efficiency through direct interaction with the environment around the system. The numerical results show that the proposed machine learning method can achieve the least mean square error in all strategies. 


\section{Introduction}

Fuel usage, energy source diversification, and electric propulsion technologies are some of the measures increasingly adopted around the world to make vehicles cleaner and more efficient with the ultimate purpose of reducing greenhouse gas emissions and reaching a sustainable energy ecosystem [1-3]. Hybrid electric vehicles are expected to have significantly lower fuel consumption than conventional vehicles as well as substantially lower emissions [4-6]. It is essential to equip hybrid electric vehicles with advanced energy management systems to achieve these goals. Electric Vehicles (EVs) and Hybrid Electric Vehicles (HEVs) can operate in a variety of modes such as fully electric or power distribution, which are controlled by an energy management system based on driving conditions [7-10]. Accordingly, an energy management system tries to improve the power output of multiple sources and supply the necessary power while minimizing the most important and significant costs $[11,12]$.

In recent years, researchers have proposed a number of models and methods for energy management in HEVs and also several reviews summarizing the progress of research in this area. Currently, the greatest challenge in improving the energy management systems of HEVs is how to shorten their computational processes and improve their adaptability. These challenges can be overcome with the help of learning methods [13-16]. In particular, reinforcement learning can be used to achieve enhanced energy management in Hybrid Energy Storage Systems (HESSs) [17, 18]. Recently, researchers have shown a growing interest in energy management approaches that utilize novel methods like machine learning or artificial intelligence [19-21]. It should be noted that machine learning is a data analyzing technique used to solve all kinds of problems in many industrial-based problems [22].

This paper presents a comprehensive approach to reinforcement learning-based energy management strategies. In general, the applications of reinforcement learning in energy management can be classified into two categories: (1) "simple algorithms," which refers to using a single algorithm (e.g., Q-learning, dynamic learning, or SARSA) to produce energy management policies; (2) "hybrid algorithms," which refers to using a combination of reinforcement learning algorithms (e.g., forecasting algorithms, deep learning algorithms, and predictive control models) or data [23].

The content of this article is organized as follows. First, the paper provides a review of HESSs and discusses the challenges of energy management in these systems and the optimization constraints in this area. Next, the paper reviews the variety of reinforcement learning methods that can be utilized in HESSs, including simple and hybrid algorithms. The article attempts to cover various methods and vehicle types and compare their main functional features.

\subsection{Hybrid Energy Storage System. A Hybrid Energy Storage} System (HESS) is an energy storage system comprised of two or more energy storage sources that meet the requirements of complex driving conditions. Considering their function, HESSs need to have a suitable energy management mechanism and topology to ensure well-coordinated power distribution among different energy sources. This also affects the lifespan of HESSs and the performance, efficiency, and cost-effectiveness of the broader system by determining how well power is distributed among different components. Energy management systems and topologies are the most popular topics of research into HESSs. Since different topologies have different power sources, there could be significant differences between different energy management systems. In recent years, many new studies have been conducted on the energy management system and topology of HESSs, but there have been few reviews of progress in this field.

The energy storage systems of EVs or HEVs have a wide range of features and functions. The performance of these systems is primarily indicated by rated power, charge/discharge rate, power density, energy density, self-discharge rate, response time, energy storage efficiency, and cycle life. With the help of these indicators, one can choose a suitable energy storage system based on performance requirements. Energy storage systems can be broadly divided into three categories of mechanical, electrical, and chemical, which are shown in Figure 1. The characteristic parameters of conventional energy storage systems are provided in Table 1.

Table 1 compares 6 energy storage systems. As can be seen, the highest power consumption is for air compressors, and the lowest is for lithium batteries. Also, the life cycle of each of these systems is presented, which can be an important criterion in choosing an energy storage system.

1.2. Energy Management Strategy. The main function of an energy management system is to balance the distribution of power between multiple energy sources and the power source so as to optimize a series of cost functions such as fuel consumption, battery life, emission, and driving control. This problem is usually formulated as a control optimization problem with specific control objectives and physical constraints. These control objectives may include one or more parameters such as exhaust temperature, emissions, fuel consumption, battery's state of charge (SOC) and state of health (SOH), and power consumption costs. Figure 2 shows the energy management problem for a typical HEV.

(1) Dynamics of power transmission systems: power transmission dynamic variables such as vehicle speed and acceleration, generator speed, power demand, battery SOC.

(2) Model and other components: basic mathematical formulations for transmission components such as electric motor, battery pack, generator, and supercapacitor, and their connections to other components.

(3) Control objectives: optimization objectives such as emission, fuel consumption, battery life, driving mobility, power costs, and shift frequency. 


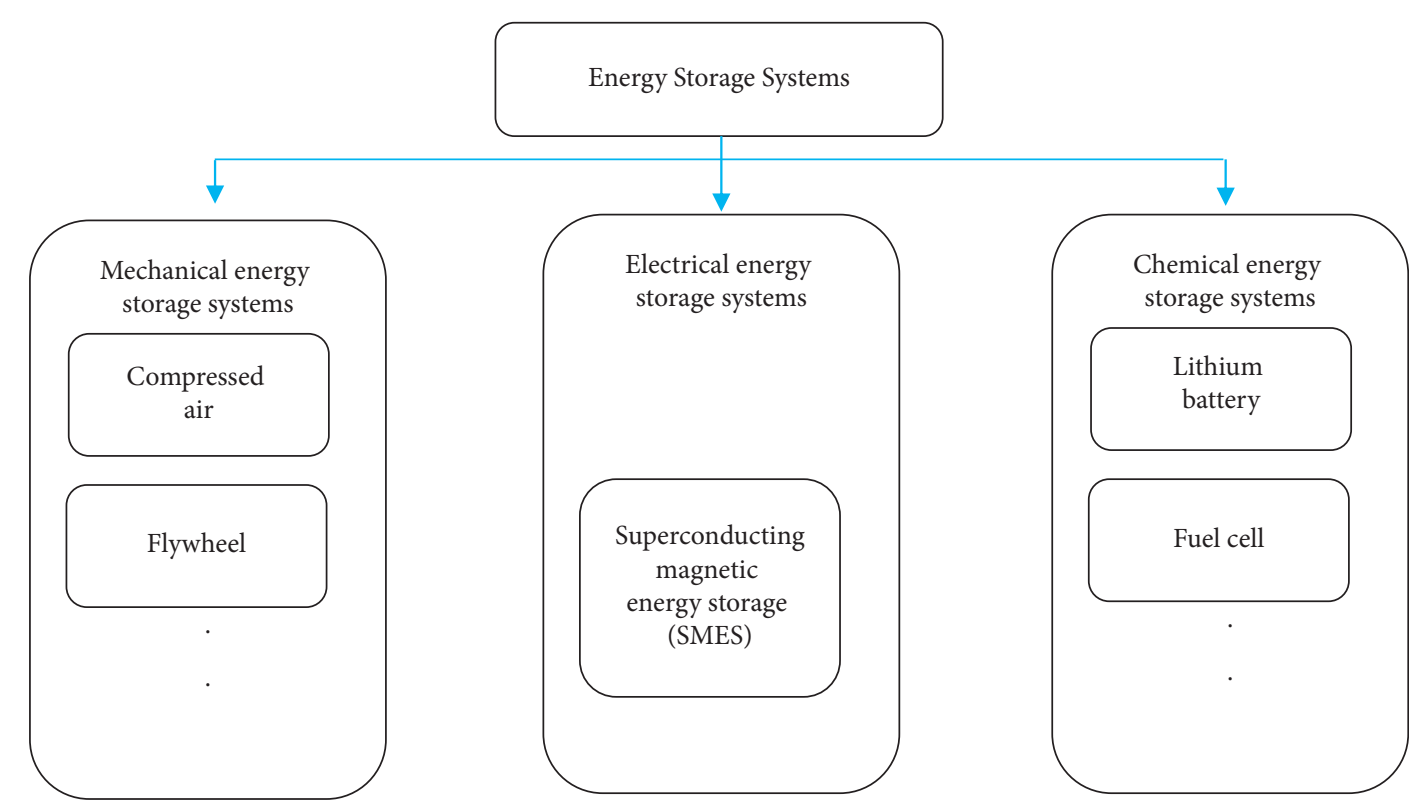

FIGURE 1: Classification of conventional energy storage systems.

TABLe 1: Typical characteristics of energy storage systems [24, 25].

\begin{tabular}{|c|c|c|c|c|c|c|c|c|c|}
\hline $\begin{array}{l}\text { Energy storage } \\
\text { systems }\end{array}$ & $\begin{array}{c}\text { Power } \\
\text { consumption } \\
\text { rate }(\mathrm{MW})\end{array}$ & $\begin{array}{l}\text { Discharge } \\
\text { time }\end{array}$ & $\begin{array}{l}\text { Power } \\
\text { density }\end{array}$ & $\begin{array}{l}\text { Energy } \\
\text { density }\end{array}$ & $\begin{array}{c}\text { Self- } \\
\text { discharge } \\
\text { rate }(\% / \text { day })\end{array}$ & $\begin{array}{l}\text { Response } \\
\text { time }\end{array}$ & $\begin{array}{c}\text { Efficiency } \\
(\%)\end{array}$ & $\begin{array}{l}\text { Lifespan } \\
\text { (years) }\end{array}$ & $\begin{array}{c}\text { Cycle life } \\
\text { (cycles) }\end{array}$ \\
\hline Compressed air & $100-300$ & 1 day & - & $30-60$ & - & minutes & $40-70$ & $20-40$ & - \\
\hline Flywheel & $0-250$ & $s-h$ & $400-1600$ & $5-130$ & $20-100$ & ms-sh & $80-90$ & $15-20$ & $10^{2}-10^{7}$ \\
\hline $\begin{array}{l}\text { Battery- } \\
\text { supercapacitor }\end{array}$ & $0-0.3$ & $\mathrm{~ms}-1 \mathrm{~h}$ & $0.1-10$ & $0.1-15$ & $2-40$ & $\mathrm{~ms}$ & $85-98$ & $5-12$ & $10^{5}-10^{6}$ \\
\hline $\begin{array}{l}\text { supercapacitor } \\
\text { SMES }\end{array}$ & $0.1-10$ & $\mathrm{~ms}-8 \mathrm{~s}$ & $500-2000$ & $0.5-5$ & $10-15$ & $\mathrm{~ms}$ & $75-80$ & - & - \\
\hline $\begin{array}{l}\text { Lithium-ion } \\
\text { batteries }\end{array}$ & $0-0.1$ & minutes-h & $200-340$ & $130-250$ & $0.1-0.3$ & $\mathrm{~ms}$ & $65-95$ & $5-8$ & $600-1200$ \\
\hline Fuel cell & $0-50$ & s-days & $\begin{array}{c}>500(\mathrm{~W} / \\
\mathrm{L})\end{array}$ & $500-3000$ & $0.5-2$ & $\begin{array}{c}\text { ms- } \\
\text { minutes }\end{array}$ & $20-66$ & $5-30$ & $10^{3}-10^{4}$ \\
\hline
\end{tabular}

(4) Physical constraints: constraints imposed on important variables such as battery SOC, power demand, rotation speed, torque, and gears.

The optimal control problem is often subject to three types of physical constraints: propulsion dynamics, initial and final values of state variables, and constraints that apply to control and state variables. Once the inputs like power demand, vehicle speed, SOC, current, steering angle, and speed are given, the amount of power needed from each energy source and the corresponding fuel cost can be calculated with the help of propulsion dynamics formulations. It is common to consider battery SOC, gearbox position, and engine/generator speed as state variables. Also, it is typical to treat engine output torque with throttle position, gear shift, and clutch position (in multimode HEVs like Toyota Prius and Chevrolet Volt) as control measures. To solve this optimal control problem, it is necessary to define appropriate constraints for these parameters. In addition, to control objectives and constraints, it is necessary to build a comprehensive model of power transmission system components as part of the solution process.

\section{Literature Review}

In reinforcement learning, an agent's core element learns how to map inputs (states/modes) to outputs (optimal control measures) to maximize a cumulative reward. This reward maximization is usually done through a trial and error process, which involves observing how each action affects the reward at present and in the future (lagged effects). The basic steps of reinforcement learning include detecting the state of the environment, taking certain actions, and improving the actions with rewards serving as guidance [26].

Reinforcement learning has three characteristic features. The first feature is the balance and coordination between exploration and exploitation. The agent uses the exploration phase to gain knowledge about the environment and then 

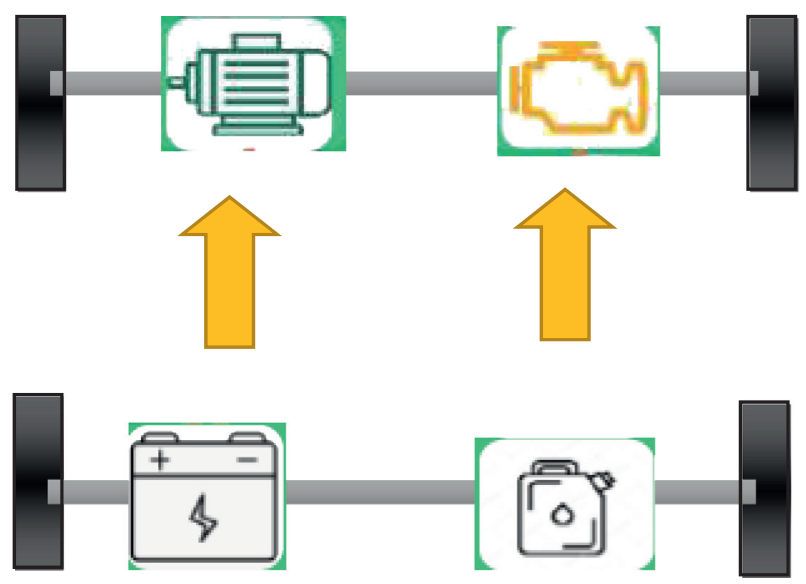

Figure 2: Classification of the energy management problem for HEVs.

proceed to exploitation, which means taking a control action based on the existing knowledge. The second feature of reinforcement learning is the ability to adapt measures without needing external control, which is essential in cases where the environment is vague or uncertain. Through its interactions with the environment, the agent can identify the state of the environment and take appropriate actions to affect it if needed. The third characteristic feature of reinforcement learning is that it is Markovian, meaning that the conditional probability distribution of future states of the environment depends only on the current state and not the sequence of events that precede it.

For the problem of energy management in HESSs, the environment can be modeled as propulsion dynamics and driving conditions. Also, the agent can be considered as a power distribution controller operating with a series of algorithms, the purpose of which is to search for a sequence of actions that maximize reward based on the available state and reward information.

To teach a reinforcement learning algorithm, it is necessary to define a value function for the agent. This value function is a function of state, action, and reward, which is typically denoted by Q(s, a) (where s is state and a is action). State, performance, and reward information can be collected in real driving conditions for the HESS energy management problem. Then, Markov decision processes (MDPs) can be used to mimic these variables, which means the next state and reward can be predicted based only on the current information and independent of historical data. Finally, the value function can be calculated to determine what will be the best control action. The difference between different reinforcement learning algorithms is in the criteria they use for updating the value function.

\subsection{Application of Reinforcement Learning in HESS Energy} Management. This section provides a summary of advanced reinforcement learning approaches used for the purpose of energy management in HESSs. The first part of the section is devoted to initial attempts to use simple algorithms in this field. Then, the section proceeds to review recent progress in the combined use of multiple algorithms and the development of hybrid algorithms for HESS energy management. Table 2 provides an overview of the variety of algorithms used in HESS energy management.

\section{Methodology}

3.1. Machine Learning. In machine learning, the subject is the design of machines that learn from the examples given to them and their own experiences. In fact, in this science, an attempt is made to design a machine using algorithms in such a way that it can learn and operate without explicitly planning and dictating each action. In machine learning, instead of programming everything, the data are given to a general algorithm, and it is this algorithm that builds its logic based on the data given to it. Machine learning has a variety of methods, including supervised, unsupervised, and reinforcement learning [2].

Machine learning is closely related to (and often overlaps with) computational statistics, the focus of which is computer prediction, and is linked to mathematical optimization, which also introduces methods, theories, and applications. Machine learning is sometimes integrated with data mining; the focus of this sub-category is on exploratory data analysis and is known as unsupervised learning. Machine learning can also be unsupervised and used to learn and recognize the basic form of the behavior of different creatures and then find significant abnormalities [3].

In data analysis, machine learning is a method for designing complex algorithms and models used for forecasting; in the industry, this is known as predictive analytics. Machine learning is beyond the realm of artificial intelligence. In the early days of artificial intelligence as a science discipline, some researchers made machines learn from data. They tried to solve this problem with various symbolic methods, like neural networks.

However, the growing emphasis on logical and knowledge-based methods has created a gap between artificial intelligence (AI) and machine learning. Probability systems were full of theoretical and practical problems with obtaining and displaying data. By 1980, cyber systems were gaining ground over AI, and statistics were no longer relevant. Work on knowledge-based learning continued within the realm of AI, leading to inductive logical programming, but the statistical trajectory of other research was beyond the realm of AI and was seen in pattern making and information retrieval. Research on neural networks was also rejected by $\mathrm{AI}$ and Computer Science (CS) at about the same time. This path was also pursued outside of AI/CS by researchers in other disciplines, including Hopfield, Rumelhart, and Hinton, under the name of connectionism. Their major success came in the mid-1980s with backpropagation [18].

Machine learning and data mining often use the same methods and overlap significantly, but while machine learning focuses on prediction based on known properties learned from training data, data mining focuses on discovering properties (formerly). The unknown focuses on the data (this is the step of analyzing knowledge extraction in the database). Data mining uses several machine learning 
TABLE 2: Comparison of reinforcement learning algorithms used in HESSs.

\begin{tabular}{|c|c|c|c|c|}
\hline Reference & Drawbacks & Advantages & Power transmission system & Algorithm \\
\hline Hsu et al. [27] & $\begin{array}{l}\text { Simplification of } \\
\text { complex models }\end{array}$ & $\begin{array}{l}\text { Adaptive to riding } \\
\text { conditions }\end{array}$ & Electric bike & Q-learning \\
\hline $\begin{array}{l}\text { Qi and Wu [28], Liu } \\
\text { and Murphey [29] }\end{array}$ & $\begin{array}{l}\text { Dependence on } \\
\text { driving data }\end{array}$ & High accuracy & Hybrid electric vehicle & Temporal-difference (TD) learning \\
\hline Liu et al. [30] & $\begin{array}{l}\text { Sporadic local } \\
\text { optimization }\end{array}$ & $\begin{array}{l}\text { Ability to run } \\
\text { online }\end{array}$ & Plug-in hybrid electric vehicle & Q-learning \\
\hline Hu et al. [31] & Computational load & $\begin{array}{l}\text { Multiple control } \\
\text { objectives }\end{array}$ & Hybrid electric vehicle & Q-learning \\
\hline Kamet et al. [32] & Design complexity & $\begin{array}{l}\text { Robust against } \\
\text { variability }\end{array}$ & Hydraulic hybrid vehicle & $\begin{array}{l}\text { Deep reinforcement learning and } \\
\text { dynamic neural programming }\end{array}$ \\
\hline $\begin{array}{l}\text { Zhao et al. [33], Xiong } \\
\text { et al. [34] }\end{array}$ & Complex mathematics & Real-time control & Hybrid truck & Dynamic learning \\
\hline $\begin{array}{l}\text { Kamet et al. [32] Zhao } \\
\text { et al. [33] }\end{array}$ & Needs specific training & Data-driven model & Plug-in hybrid electric vehicle & Deep learning \\
\hline $\begin{array}{l}\text { Liu et al. [30], Hu } \\
\text { et al. [31] }\end{array}$ & $\begin{array}{l}\text { Sensitivity to driving } \\
\text { cycle }\end{array}$ & Fast computation & Electric vehicle & Online reinforcement learning \\
\hline $\begin{array}{l}\text { Hay et al. [35], Lin } \\
\text { et al. [36] }\end{array}$ & Data requirements & $\begin{array}{l}\text { Improved battery } \\
\text { life }\end{array}$ & $\begin{array}{l}\text { Hybrid electric vehicle and } \\
\text { plug-in hybrid electric vehicle }\end{array}$ & $\begin{array}{l}\text { Reinforcement learning and } \\
\text { Markov decision system }\end{array}$ \\
\hline
\end{tabular}

methods but with different purposes; machine learning, on the other hand, uses data mining techniques as "unsupervised learning" or as a preprocessing step to improve learner accuracy. Much of the confusion between the two disciplines (often with distinct conferences and journals, with the exception of the ECML PKDD) stems from their underlying assumptions: In machine learning, performance is usually assessed by the ability to reproduce known knowledge, while In knowledge extraction and data mining (KDD), the key activity is to discover previously unknown knowledge. Compared to known knowledge, one unsupervised method (an uninformed method) easily fails other monitored methods, while in a typical KDD activity, supervised methods can be accessed due to lack of access to training data.

Classification machine learning models can be accurately estimated using techniques such as the holdout method, which divides data into a training set and an experimental set (usually two-thirds of the data in the training set and one-third). Moreover, it evaluates the model's performance under training on the test set verified. In comparison, the $\mathrm{N}$-fold cross-validation method randomly divides the data into $k$ subsets, with $k-1$ of the data used for model training and $k-1$ for testing the model's predictive ability. In addition to holdout and cross-validation methods, bootstrap, which samples $n$ items from the data set by pasting, can be used to evaluate the model accurately [18].

3.2. Learning Algorithms. In 2012, Hsu et al. [27] used the Q-learning algorithm to improve the power management system of electric or hybrid bikes. These researchers defined the power management objectives as improvement in riding safety and comfort and more efficient use of battery power. The simulation results of this study showed that the proposed power management system could offer $24 \%$ and $50 \%$ improvement in the riding quality and energy efficiency objectives, respectively. Since then, many researchers have started using reinforcement learning algorithms in HESS energy management rather than the control optimization theory. For example, Qi et al. used the Q-learning algorithm to optimize the battery SOC maintenance strategy of an HEV [28]. When combined with a sustainable strategy, this method can offer a balance between real-time performance and energy efficiency optimization. In [29], Liu used the reverse reinforcement learning method to create a probabilistic driving path prediction system, which predicts the suitable engine/battery power distribution ratio based on its forecast of driver behavior.

Over the past several years, Liu et al. have also conducted a number of studies on the use of reinforcement learningbased power distribution controls in hybrid transmission systems. First, they evaluated the adaptability, optimality, and learning capability of a Q-learning-based energy management strategy for a hybrid tracked vehicle [30]. Next, to develop real-time controls for a hybrid transmission system, they integrated an online recursive algorithm into the Q-learning structure so that control strategies can be updated in real-time [31]. However, these algorithms may not be robust against variability in driving conditions, i.e. different driving behaviors, driving areas, and road environments.

\section{Proposed Hybrid Energy System}

With the rapid development of deep learning and artificial intelligence in recent years, the energy management strategies of hybrid vehicles have become increasingly intelligent. It is now typical to embed two or more algorithms that process different types of information into a reinforcement learning framework in order to ensure more efficient, realtime controls based on speed and power requirement forecasts; information shared between vehicles or between vehicles and infrastructure, and interactions with smart grids and smart cities. 


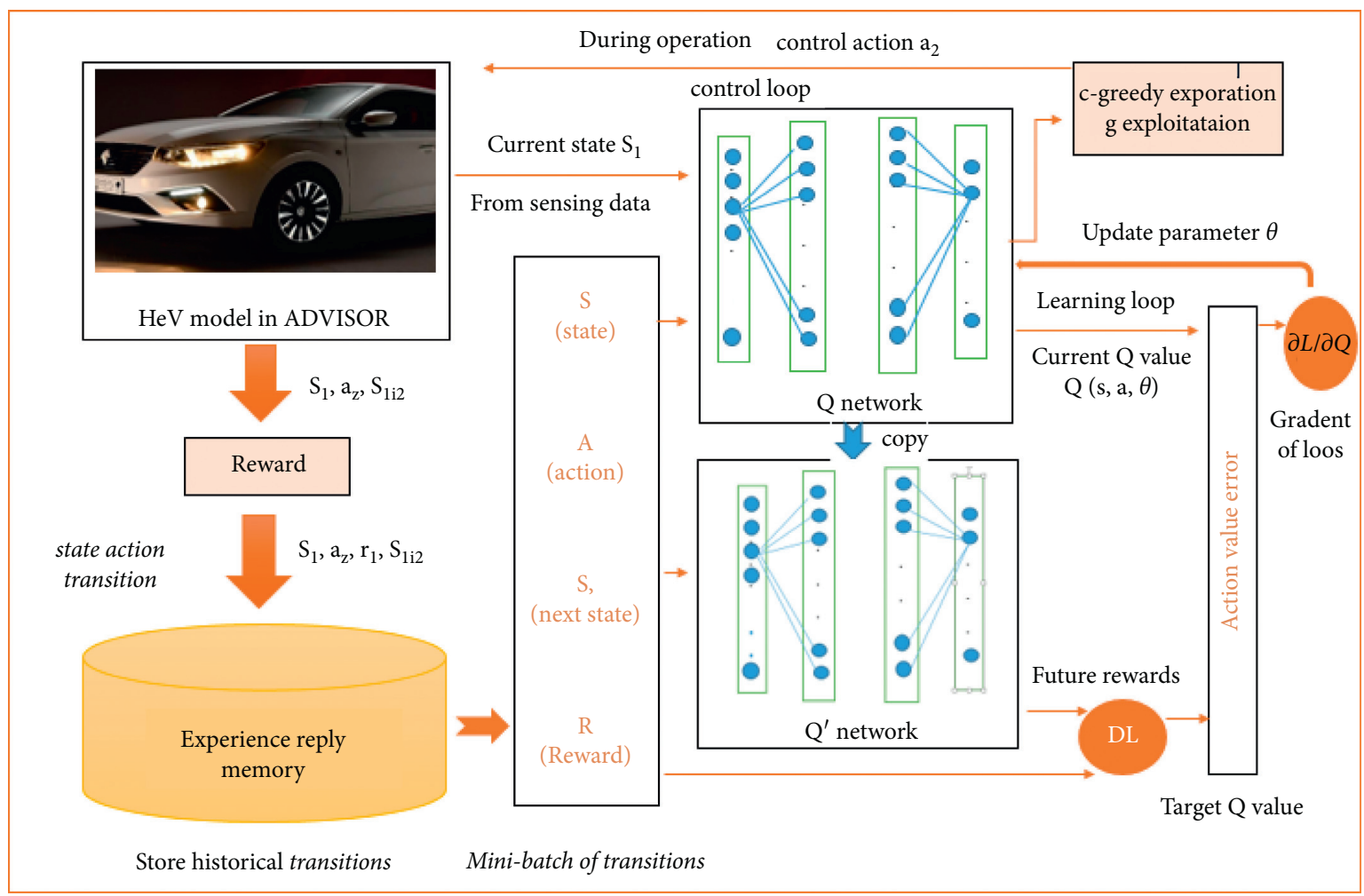

FIGURE 3: DRL-based control framework of [12] as an example of hybrid algorithms developed for energy management in HEVs.

TABLE 3: Machine learning implementation results for HESSs.

\begin{tabular}{|c|c|c|c|c|c|c|c|}
\hline \multirow[t]{2}{*}{ Strategy } & \multicolumn{2}{|c|}{$\begin{array}{c}\text { Without } \\
\text { transactions }\end{array}$} & \multicolumn{2}{|c|}{ With transactions } & \multirow[t]{2}{*}{ Gradient of loss } & \multirow[t]{2}{*}{ Action error } & \multirow[t]{2}{*}{ Mean square error } \\
\hline & Train & Test & Train & Test & & & \\
\hline 1 & 1500 & 500 & 1300 & 700 & 0.376 & 0.305 & 0.343 \\
\hline 2 & 1700 & 300 & 1000 & 1000 & 0.360 & 0.450 & 0.303 \\
\hline 3 & 1000 & 1000 & 1700 & 300 & 0.424 & 0.403 & 0.334 \\
\hline 4 & 1300 & 700 & 1500 & 500 & 0.391 & 0.330 & 0.476 \\
\hline 5 & 1500 & 500 & 1500 & 500 & 0.396 & 0.311 & 0.259 \\
\hline
\end{tabular}

Deep reinforcement learning (DRL) has also proved to be an effective tool for designing an adaptive energy management strategy based on driving cycle data. In a study by $\mathrm{Hu}$ et al., they evaluated the performance of a DRL-based energy management strategy with online learning capability in comparison to a rule-based strategy [32]. The diagram of the DRL-based control strategy proposed in this study is illustrated in Figure 3. Moreover, the provided numerical results are illustrated in Table 3 and Figure 4.

According to Figure 3, there are two neural networks, a neural network for the nontransaction mode and a neural network for the transaction mode. Accordingly, Table 3 presents five different strategies for machine learning. In each strategy, the amount of data separation into two groups Train and Test (for each neural network) is specified. Also, three error indicators are presented after the implementation of the machine learning approach. The results in Table 3 and Figure 4 show that strategy 5 had the lowest error rate.
This is because, in strategy 5, both neural networks are implemented in a similar way. Moreover, the highest error was reported for strategy 2 , in which the data were evenly divided into two groups, train and test. Accordingly, it can be concluded that the proposed machine learning method can achieve the least mean square error in all strategies.

Real-time management under driving conditions must be achieved through the selection of the best adaptive strategies based on changes in the value function. In the case of hybrid algorithms, more data are needed for this purpose. In [33], researchers used a deep neural network (DNN) to train value functions offline and then used these functions in Q-learning for online control with adaptability to different transmission systems and driving conditions.

Xiong et al. developed a hybrid reinforcement learningbased real-time energy management system by combining the Q-learning algorithm with an online value function updating procedure. The product of this combination was a 


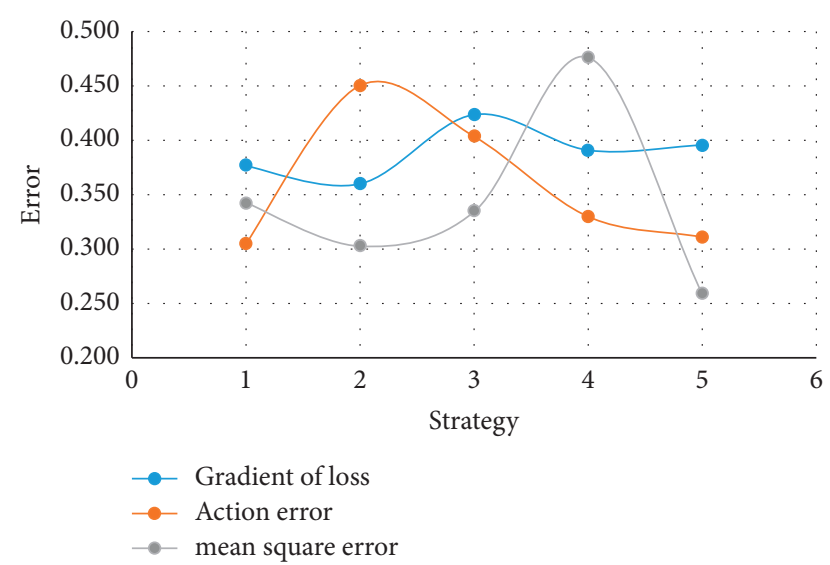

FIgURE 4: Different error indexes for all strategies.

real-time control system, where control measures can be updated in real time. They then validated this strategy by running simulations on a hardware operating system with batteries and super capacitors added to the circuit [34].

\section{Conclusion}

In machine learning, it is a broad discipline that has designed learning algorithms that can guide stimuli, detect spoken language, and discover hidden settings in data volume growth. Financial data are no exception. It works with data streams that capture company characteristics, corporate governance characteristics, audit reports, market data, and environmental variables. Machine learning algorithms detect complex patterns in this data, select the best variables to explain the variable, and use the appropriate combination of variables to predict the sample accurately. They are the keys to opening up big, growing data sources that can make better predictions and make smarter decisions. Machine learning has received a great deal of attention in the social sciences and denies the existing approaches to data analysis.

Although deep learning-based energy management strategies are superior to rule-based strategies, two issues limit their use in practice. The first issue is the limited computation power of the vehicle's CPU, which makes it necessary to install another computer on the vehicle to process the data. The second issue is collecting and storing the required information because deep learning requires substantial amounts of data to infer strategies for different driving conditions. However, with the development of network communication technologies and intelligent transportation systems (ITS), it is likely to become easier to use deep learning-based real-time energy management strategies in the future.

This paper provided a summary of reinforcement learning-based energy management strategies for Hybrid Energy Storage Systems (HESSs) of hybrid electric vehicles (HEVs). The paper started with an introduction to the problem of energy management in this field and how learning methods can be used to solve this problem. Then, the existing energy management schemes with multiple control objectives were discussed. In the end, the outlook for the further development of reinforcement learning-based energy management systems is as described.

A potentially rewarding line of research in this field is to work on the more efficient use of artificial intelligence techniques for energy management. It is also important to assess the theoretical and practical feasibility of the proposed methods through real-world testing and implementation as well as simulation. Considering the advancement of intelligent transportation systems, which also make it easier to gather traffic data, it might also be helpful to develop a method for adjusting strategies based on the behaviors of vehicles and infrastructure.

\section{Data Availability}

The data are available from the corresponding author on request.

\section{Conflicts of Interest}

The authors declare that they have no conflicts of interest.

\section{References}

[1] F. Arslan, "A review of machine learning models for software cost estimation," Review of Computer Engineering Research, vol. 6, no. 2, pp. 64-75, 2019.

[2] A. Manishimwe, H. Alexander, H. Kaluuma, and M. Dida, "Integrated mobile application based on machine learning for East Africa stock market," Journal of Information Systems Engineering \& Management, vol. 6, no. 3, Article ID em0143, 2021.

[3] L. H. Salazar, A. Fernandes, R. Dazzi, N. Garcia, and V. R. Leithardt, "Using different models of machine learning to predict attendance at medical appointments," Journal of Information Systems Engineering and Management, vol. 5, no. 4, Article ID em0122, 2020.

[4] R. Gaussmann, D. Coelho, A. Fernandes, P. Crocker, and V. R. Q. Leithardt, "Estimated maintenance costs of Brazilian highways using machine learning algorithms," Journal of Information Systems Engineering and Management, vol. 5, no. 3, Article ID em0119, 2020.

[5] Y.-P. Xu, P. Ouyang, S.-M. Xing, L.-Y. Qi, M. khayatnezhad, and H. Jafari, "Optimal structure design of a PV/FC HRES using amended Water Strider Algorithm," Energy Reports, vol. 7, pp. 2057-2067, 2021.

[6] A. Ma, J. Ji, and M. Khayatnezhad, "Risk-constrained nonprobabilistic scheduling of coordinated power-to-gas conversion facility and natural gas storage in power and gas based energy systems," Sustainable Energy, Grids and Networks, vol. 26, Article ID 100478, 2021.

[7] R. A. Salas-Rueda, "Analysis on the use of continuous improvement, technology and flipped classroom in the teachinglearning process by means of data science," The Online Journal of Communication and Media Technologies, vol. 8, no. 4, pp. 325-343, 2018.

[8] C. Dépature, S. Jemei, L. Boulon et al., "Energy management in fuel-cell $\backslash$ /battery vehicles: key issues identified in the IEEE vehicular technology society motor vehicle challenge 2017," IEEE Vehicular Technology Magazine, vol. 13, no. 3, pp. 144-151, 2018.

[9] B. Subiyakto and S. Kot, "The government reform on healthcare facilities from the standpoint of service quality 
performance," International Journal of Economics and Finance Studies, vol. 12, no. 1, pp. 16-31, 2020.

[10] A. Mostafaeipour, M. Qolipour, H. Goudarzi et al., "Implementation of adaptive neuro-fuzzy inference system (ANFIS) for performance prediction of fuel cell parameters," Journal of Renewable Energy and Environment, vol. 6, no. 3, pp. 7-15, 2019.

[11] L. Mapfumo and S. Mutereko, "Contextualising stakeholder participation in the governance of Harare's informal economy sector," International Journal of Economics and Finance Studies, vol. 12, no. 1, pp. 103-118, 2020.

[12] A. J. De Bruyn, "Harnessing Hr governance in effective virtual teams," International Journal of Social Sciences and Humanity Studies, vol. 12, no. 1, pp. 1-17, 2020.

[13] A. Mostafaeipour, A. Goli, and M. Qolipour, "Prediction of air travel demand using a hybrid artificial neural network (ANN) with Bat and Firefly algorithms: a case study," The Journal of Supercomputing, vol. 74, no. 10, pp. 5461-5484, 2018.

[14] N. E. L. Danielle and L. Masilela, "Open governance for improved service delivery innovation in South Africa," International Journal of eBusiness and eGovernment Studies, vol. 12 , no. 1, pp. 33-47, 2020.

[15] K. Mosala and E. Chinomona, "Motorists attitudes towards implementation of E-tolls in Gauteng Province, South Africa," International Journal of eBusiness and eGovernment Studies, vol. 12, no. 1, pp. 48-62, 2020.

[16] A. H. Rasouli Amirabadi and M. Mirzaei, "Photosensitization of coronene-purine hybrids for photodynamic therapy," Quarterly Journal of Iranian Chemical Communication, vol. 7, no. 4, pp. 352-471, 2019.

[17] A. El-Khateeb, "Practical biochemistry principles and techniques approach," Progress in Chemical and Biochemical Research, vol. 3, no. 3, pp. 180-193, 2020.

[18] I. H. Sarker, "Machine learning: algorithms, real-world applications and research directions," SN Computer Science, vol. 2, no. 3, pp. 1-21, 2021.

[19] A. Rahimian Boogar, A. Gholamalizadeh Ahangar, and E. Shirmohamadi, "Efficiency of foliar application of humic acid on improve absorb of $\mathrm{K}$ than $\mathrm{Na}$ and salt tolerance in petunia hybrida L," International Journal of Advanced Biological and Biomedical Research, vol. 2, no. 42, pp. 256-259.

[20] M. Rahimi, S. Jamehbozorgi, H. Chermette, R. Ghiasi, and M. Poor Kalhor, "Computational study of substituent effect on the electronic properties of ferrocylidene acetophenones complexes," Eurasian Chemical Communications, vol. 1, no. 5, pp. 411-418, 2019.

[21] Y. Wang, X. Wu, B. Shao, X. Yang, G. Owens, and H. Xu, "Boosting solar steam generation by structure enhanced energy management," Science Bulletin, vol. 65, no. 16, pp. 1380-1388, 2020.

[22] W. Bi, Y. Shu, W. Dong, and Q. Yang, "Real-time energy management of microgrid using reinforcement learning," in Proceedings of the 2020 19th International Symposium on Distributed Computing and Applications for Business Engineering and Science (DCABES), pp. 38-41, IEEE, Xuzhou, China, October 2020.

[23] Z. Yang, F. Wu, and J. Zhao, "A survey: limited data problem and strategy of reinforcement learning," in Proceedings of the 2021 Chinese Intelligent Automation Conference, Springer, Singapore, pp. 471-481, 2022.

[24] A. Chatzivasileiadi, E. Ampatzi, and I. Knight, "Characteristics of electrical energy storage technologies and their applications in buildings," Renewable and Sustainable Energy Reviews, vol. 25, pp. 814-830, 2013.
[25] O. Z. Sharaf and M. F. Orhan, "An overview of fuel cell technology: fundamentals and applications," Renewable and Sustainable Energy Reviews, vol. 32, pp. 810-853, 2014.

[26] R. S. Sutton and A. G. Barto, Reinforcement Learning: An Introduction, MIT Press, London UK, 2018.

[27] R. C. Hsu, C. T. Liu, and D. Y. Chan, "A reinforcementlearning-based assisted power management with QoR provisioning for human-electric hybrid bicycle," IEEE Transactions on Industrial Electronics, vol. 59, no. 8, pp. 3350-3359, 2011.

[28] X. Qi, G. Wu, K. Boriboonsomsin, M. J. Barth, and J. Gonder, "Data-driven reinforcement learning-based real-time energy management system for plug-in hybrid electric vehicles," Transportation Research Record: Journal of the Transportation Research Board, vol. 2572, no. 1, pp. 1-8, 2016.

[29] C. Liu and Y. L. Murphey, "Power management for plug-in hybrid electric vehicles using reinforcement learning with trip information," in Proceedings of the 2014 IEEE Transportation Electrification Conference and Expo (ITEC), pp. 1-6, IEEE, Dearborn, MI, USA, June 2014.

[30] T. Liu, Y. Zou, D. Liu, and F. Sun, "Reinforcement learning of adaptive energy management with transition probability for a hybrid electric tracked vehicle," IEEE Transactions on Industrial Electronics, vol. 62, no. 12, pp. 7837-7846, 2015.

[31] J. Hu, D. Liu, C. Du, F. Yan, and C. Lv, "Intelligent energy management strategy of hybrid energy storage system for electric vehicle based on driving pattern recognition," Energy, vol. 198, p. 117298, 2020.

[32] A. A. Kamel, H. Rezk, and M. A. Abdelkareem, "Enhancing the operation of fuel cell-photovoltaic-battery-supercapacitor renewable system through a hybrid energy management strategy," International Journal of Hydrogen Energy, vol. 46, no. 8, pp. 6061-6075, 2021.

[33] P. Zhao, Y. Wang, N. Chang, Q. Zhu, and X. Lin, “A deep reinforcement learning framework for optimizing fuel economy of hybrid electric vehicles," in Proceedings of the 2018 23rd Asia and South Pacific design automation conference (ASP-DAC), pp. 196-202, IEEE, Jeju, South Korea, January 2018.

[34] R. Xiong, J. Cao, and Q. Yu, "Reinforcement learning-based real-time power management for hybrid energy storage system in the plug-in hybrid electric vehicle," Applied Energy, vol. 211, pp. 538-548, 2018.

[35] H. Yu, M. Kuang, and R. McGee, "Trip-oriented energy management control strategy for plug-in hybrid electric vehicles," in Proceedings of the 2011 50th IEEE Conference on Decision and Control and European Control Conference, pp. 1323-1336, Orlando, FL, USA, January 2013.

[36] X. Lin, Z. Wang, and J. Wu, "Energy management strategy based on velocity prediction using back propagation neural network for a plug-in fuel cell electric vehicle," International Journal of Energy Research, vol. 45, no. 2, pp. 2629-2643, 2021. 